\title{
EXTRA CORPOREAL SHOCK WAVE - A NEW WAVE OF THERAPY
}

Eva Goyal, Guljot Singh ${ }^{2}$, Vivek Sharma ${ }^{3}$, Jaspreet Singh Gill ${ }^{4}$, Gagandeep Gupta ${ }^{5}$

${ }^{1}$ PG Student, Department Of Periodontics, Desh Bhagat Dental College \& Hospital, Punjab, India

${ }^{2}$ HOD \& Professor, Department Of Periodontics, Desh Bhagat Dental College \& Hospital, Punjab, India

${ }^{3}$ Professor, Department Of Periodontics, Desh Bhagat Dental College \& Hospital, Punjab, India

${ }^{4}$ Reader, Department Of Periodontics, Desh Bhagat Dental College \& Hospital, Punjab, India

${ }^{5}$ Senior Lecturer, Department Of Periodontics, Desh Bhagat Dental College \& Hospital, Punjab, India

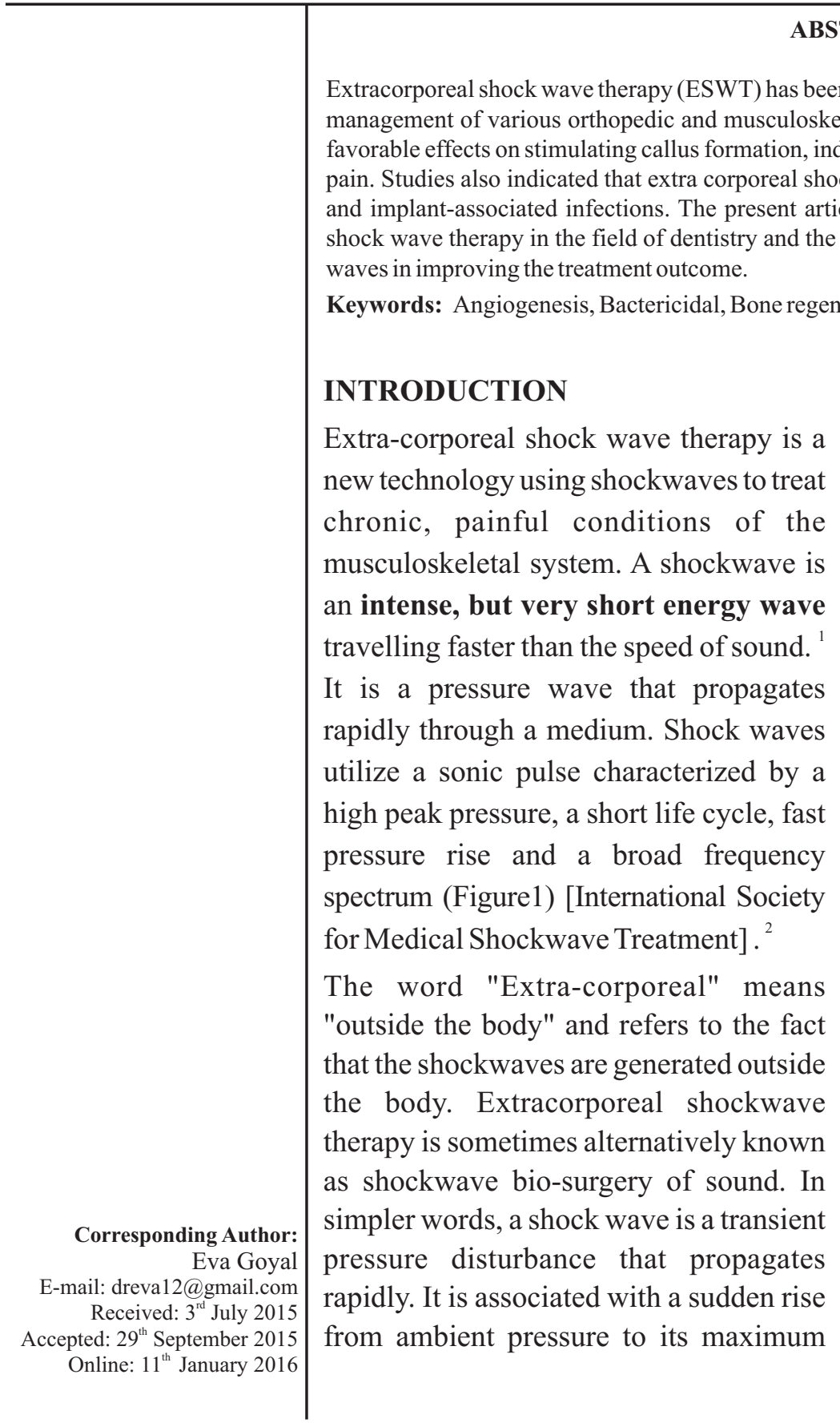

\section{ABSTRACT}

Extracorporeal shock wave therapy (ESWT) has been enormously used in medical practice, especially for the management of various orthopedic and musculoskeletal disorders. Extracorporeal shock wave therapy has ind incing angiogenesis and bone regeneration and relieving

Extra-corporeal shock wave therapy is a chronic, painful conditions of the musculoskeletal system. A shockwave is an intense, but very short energy wave travelling faster than the speed of sound. It is a pressure wave that propagates utilize a sonic pulse characterized by a high peak pressure, a short life cycle, fast pressure rise and a broad frequency spectrum (Figure1) [International Society for Medical Shockwave Treatment].

\section{"outside the body" and refers to the fact} that the shockwaves are generated outside the body. Extracorporeal shockwave therapy is sometimes alternatively known as shockwave bio-surgery of sound. In simpler words, a shock wave is a transient pressure disturbance that propagates rapidly. It is associated with a sudden rise

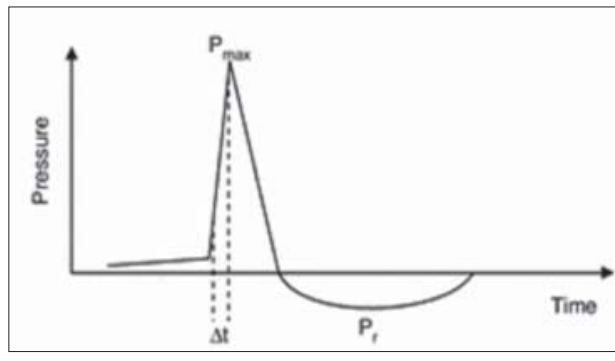

Figure 1. Characteristics of shockwaves. $\mathrm{P}_{\max }=$ pressure maximum; $\mathrm{P}_{1}=$ negative peak pressure; $\Delta \mathrm{t}=$ pressure rise time

pressure. The machine used allows controlling and focusing the shockwaves to such an extent that we are able to pass the shockwaves through the uninjured portions of the body without any effect, and deliver the energy to a focus point at the level of the injured tissue. Initially, this shockwave exerts a mechanical pressure and tension force on the afflicted tissue. This has been shown to create an increase in cell membrane permeability, thereby increasing microscopic circulation to the tissues and the metabolism within the treated tissues, both of which promote healing and 
subsequent dissolution of pathological calcific deposits. Next, the extra corporeal shock wave therapy shock waves pressure front creates "cavitation bubbles" behind it. Cavitation bubbles ( Figure 2) are simply small empty spaces created behind the energy front. They tend to expand to a maximum size, then collapse, much like a bubble popping. As these bubbles burst, a resultant force is created. In the human body, this force is strong enough to help break down pathological deposits of calcification in soft tissues. ${ }^{3}$

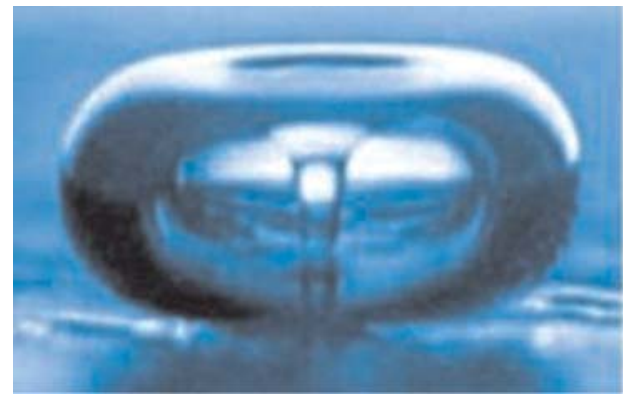

Figure 2: Cavitation bubble

Then, as cavitation bubbles collapse, they create smaller, secondary energy waves known as "micro jets" (Figure 3). These micro jets also create a lot of force that also breaks down pathological deposits of calcification in the soft tissues through direct, mechanical means. ${ }^{3}$

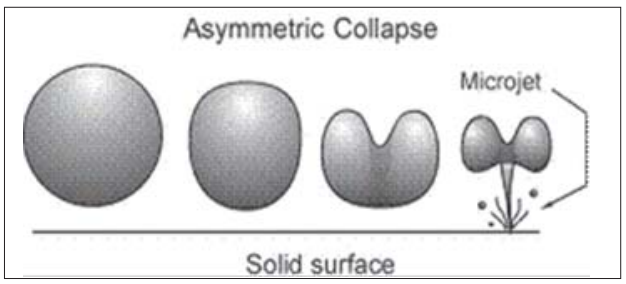

Figure 3: Micro jets are created by the collapse of cavitation bubbles

In the application of an extra corporeal shock wave treatment in a medical setting, however, it's not just one cavitation bubble or just a few cavitation bubbles being produced, but hundreds and thousands (Figure 4).

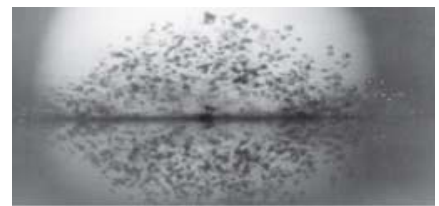

Figure 4: Hundreds of cavitation bubbles being produced

\section{HISTORICALBACKGROUND}

Extracorporeal shock wave therapy was introduced in Germany in 1980s. It has been used for the management of urolithiasis, cholelithiasis and sialolithiasis. ${ }^{3}$ The principle was then extended to a wide range of treatment modalities like treatment of dermal wounds, orthopedic conditions and musculoskeletal conditions. ${ }^{3}$

Extra corporeal shock wave therapy was also observed to have a good clinical effect on pain. Specialized machines were then developed specifically with the idea of using these shockwaves on other parts of the body, and this is the origin of extra corporeal shockwave therapy. There appears a vast scope in the treatment of oral and maxillofacial conditions. ${ }^{3,4,5,6}$

\section{CLINICAL APPLICATIONS}

\section{Energy from Shock waves and Cavitation}

Sialolithiasis is one of the common conditions affecting the salivary glands. It refers to the 'calcified mass' that forms in the salivary gland, usually the Wharton's duct of the submandibular gland. Surgical procedures involving incision of the salivary gland duct followed by removal of the stone and/or sialo-adenectomy are commonly undertaken, carrying the risks of damage to the vital structures in the vicinity. ${ }^{1}$ However, as an alternative, Extra corporeal shock wave therapy has been employed, especially in situations where the size of the calculi do not exceed 7 millimeter. ${ }^{5}$ The pressure pulses of the shock wave release shearing forces and cavitation energy that cause disintegration of the calculi. Hence, they can prove to be a safe, effective, minimally-invasive, non-surgical treatment option for sialolithiasis. $^{5}$

\section{Role in calculus removal}

A study was conducted by Muller in 2003 to explore the possibility of utilizing shock waves for dental calculus and biofilm removal and hence, in the treatment of periodontitis. The authors modified the shock wave device and designed a hand piece that could simulate the techniques that are employed regularly for calculus and biofilm removal. The study concluded that Extra corporeal shockwave therapy had a reduced efficiency in calculus removal when compared to an ultrasonic 
instrument. ${ }^{7}$ However; the efficiency for biofilm removal was comparable with an ultrasonic instrument. Further improvements in extra corporeal shockwave therapy equipment can provide a good treatment option for calculus removal and imparting an antibacterial action and disruption of bacterial biofilms ${ }^{1}$ at the same time, thus proving to be having a superior edge over conventional scalers to control periodontitis.

\section{Role in Inflammation}

Although, the biochemical mechanisms underlying the anti-inflammatory effect of extra corporeal shockwave treatment are not fully understood, some reports present data indicating the critical role played by nitric oxide in this therapeutic effect. One of the possible molecular mechanisms of action behind the anti-inflammatory effect of extra corporeal shock wave therapy seems to be their capacity to keep local nitric oxide contents at a physiological level in the early phase of inflammatory response, enhancing either a non-enzymatic or enzymatic production of nitric oxide. ${ }^{8}$ The principal clinical observation after extra corporeal shock wave treatment was the immediate increase in blood flow around the treated area. ${ }^{9}$ Extra corporeal shockwave therapy, therefore, may in future be applied in an increasing number of inflammatory diseases for which there is presently no effective therapy.

\section{Implications in Dentistry}

Effect of extra corporeal shockwave therapy on inflammation can also be explored more to be used in various dental specialties. The application of extra corporeal shockwave therapy during orthodontic treatment showed that shock waves induce increased Interleukin- $1 \beta$ production as part of mechanical forces transduction, thus triggering a biologic response. This in turn could contribute to accelerate periodontal remodeling and increased periodontal stability of the orthodontically aligned teeth, hence foreshortening of the orthodontic tooth movement period and the relapse risk. Orthodontic force application triggers an inflammatory reaction in the periodontal tissue surrounding the involved teeth, resulting in tooth movement. Preliminary work revealed that extracorporeal shock wave therapy increased the expression of the inflammatory cytokines involved.

Hazan-Molina H, et al fabricated orthodontic appliance and applied between the molars and the incisors of adult Wistar rats. In conjunction with orthodontic force commencement, the rats were treated with a single episode of 1000 shock waves. The rats were killed and interleukin (IL)- $1 \beta$, Interleukin -6 and tumour necrosis factor-alpha were evaluated. The percentage of the area staining positively for all inflammatory cytokines during the first 2 days decreased statistically significantly more in the shock wave-treated group compared with the non-treated control group.

The documented evidence on the use of extra corporeal shockwave therapy in periodontics, however, is very limited. Periodontitis is an immunoinflammatory disease that leads to destruction of periodontal ligament and adjacent supporting alveolar bone. It is caused by pathogenic sub-gingival microbial biofilms containing several periodontal pathogens. ${ }^{10}$

\section{Bactericidal effects}

The use of extra corporeal shockwave therapy in destruction of bacteria has been a question of debate. Various studies conducted to evaluate the bactericidal efficacy of shock waves in destruction of bacteria have given controversial results. One such study showed that shock waves at high energy levels have a lethal effect on bacteria ${ }^{8}$, while other studies concluded that the microorganisms continued to persist and cause inflammation in the sites treated with shock wave.

\section{Oral Micro-organisms}

A study conducted in 2008 by Novak et al considering the efficacy of shock waves on oral bacteria especially periodonto- ${ }^{-}$athogens reported that shock waves at low doses of 100 pulses at $0.3 \mathrm{~mJ} / \mathrm{mm} 2$ had a selective bactericidal effect on two commonly detected bacterial species implicated in periodontal pathologies, particularly Streptococcus mutans and an unencapsulated strain of Porphyromonas gingivalis. ${ }^{11}$ It also showed that a significant disruption of oral bacterial aggregates occurred with these low energy levels of shock waves and that the viability of other microorganisms was not significantly affected by it. ${ }^{11}$ Similar results on disruption of bacterial biofilms from 
tooth surface have also been reported wherein the potential of shock waves was at par comparison levels with ultrasonic instruments. ${ }^{12}$ Extra corporeal shockwave therapy could break up the biofilm layers and disperse individual bacteria into surrounding tissues, leading to increased susceptibility to antibacterial agents and the access of antibiotics and inflammatory cells to avascular areas could be improved by neovascularization and tissue regeneration. ${ }^{13}$ Furthermore, these studies concluded that infections should no longer be generally classified as a contraindication for shock-wave treatment.

\section{Bone Regeneration}

\section{Orthopedics and Oral Surgery}

Application of extra corporeal shockwave therapy in induction of bone regeneration is frequently used in orthopedic diseases to induce bone healing in cases of non-union, delayed union and bone necrosis. ${ }^{14,15,16}$ Shock waves have a potential to induce bone regeneration with energy levels of $0.16 \mathrm{~mJ} / \mathrm{mm}^{2}$ in a range between 250 and 500 impulses. Following the treatment of the orthopedic sites with shock waves, it was noted that there was an increased expression of osteogenic markers like bone morphogenic proteins $(\mathrm{BMP})^{6}$, transforming growth factor $(\mathrm{TGF}-\beta),{ }^{17}$ increase of bone alkaline phosphatase activity ${ }^{5}$ and osteocalcin mRNA expression. ${ }^{5}$ This property of bone regeneration of extra corporeal shock waves was also extended for improved healing of experimentally induced mandibular sub-condylar fractures, where it significantly improved the fracture healing. Hence, it was concluded that extra corporeal shockwave therapy combined with inter-maxillary fixation can be an effective therapy for accelerated fracture healing and even for reduction in the incidence of complications associated with fracture healing. ${ }^{17}$

\section{Periodontics}

The potential of shock waves to activate osteogenic growth factors and in the induction of new bone formation has been successfully applied and extra corporeal shockwave therapy has proved to successfully promote regeneration of alveolar bone lost, following experimentally induced periodontal disease. $^{18}$ In addition, the degree of regeneration improved in accordance to the dose of extra corporeal shockwave therapy applied. Also, the regenerative effect of extra corporeal shockwave therapy seemed to last for a longer period with higher doses of 300 and 1000 pulses at energy flux density (EFD) of 0.1 $\mathrm{mJ} / \mathrm{mm}^{2}{ }^{18}$ Hence, there exists a scope for further studies to pave the way for future developments in this field of periodontal regeneration, where extra corporeal shockwave therapy could prove to be a valuable adjunct.

Extracorporeal shock wave therapy (ESWT) could promote the regeneration of alveolar bone following Porphyromonas gingivalis induced periodontitis in rats. Sathish kumar S et al in 2008 conducted a study where rats were infected with $P$. gingivalis for 10 weeks, which caused alveolar bone resorption. The rats were then treated with a single episode of 100, 300, or 1000 impulses of shock wave on both cheeks at energy levels $0.1 \mathrm{~mJ} / \mathrm{mm} 2$. Alveolar bone levels were determined at $0,3,6$, and 12 weeks following extra corporeal shockwave therapy and compared with those in untreated controls. Infected rats treated with 300 and 1000 impulses demonstrated significantly improved alveolar bone levels at 3 weeks compared with untreated controls, and the improved levels remained for at least 6 weeks in most rats. The results of these studies have demonstrated effective regeneration of alveolar bone by extra corporeal shockwave therapy and suggest that extra corporeal shockwave therapy should be evaluated as an adjunct in the regeneration of periodontal tissues following periodontal disease. $^{7}$

\section{Management of Peri implantitis}

Dental implants have been widely used clinically in recent decades, but peri-implantitis is still a common complication of dental implants with high incidence, which is greatly harmful to the longevity of the dental implant. The current treatment of periimplantitis is limited and it is hard to achieve optimal re-osseointegration. Extra corporeal shockwave therapy has favorable effects on treating delayed union and non-union of fracture, promoting fracture healing, and inducing bone regeneration. Studies indicated that extracorporeal shock waves may activate osteoblasts 
and their precursors, and has a bactericidal effect on several oral pathogens. Hence, extra corporeal shockwave therapy may be an adjuvant treatment for peri-implantitis by controlling infection, inducing alveolar bone regeneration and promoting reosseointegration. . $^{19,20}$

\section{CONCLUSION}

The advantages of extra corporeal shockwave therapy are non-invasiveness and low rate of complications. Primary aim should be to evaluate adequate energy density levels and impulse rates for specific groups of indications using high quality studies according to evidence-based-medicine. Although we do not have sufficient literature available, extra corporeal shockwave therapy has shown promising results and can be implemented in periodontal therapy. Its significant bactericidal effect to the periodontopathogens, tendency to remove calculus, potential to induce alveolar bone and tissue regeneration can be a boon for periodontal therapy. In simple words, it is a non-invasive therapy that could avoid repeated surgeries such as debridement, shorten the rehabilitation time, promote early function training and reduce the costs.

However, it still remains unclear whether extra corporeal shockwave therapy has direct favourable effects on the immune response. Extra corporeal shockwave theraspy is contraindicated in pregnancy and for people on medications that interfere with blood clotting such as Coumadin and prophylactic aspirin. Infected target areas are still considered as a contraindication for extra corporeal shockwave therapy because of the risk of bacterial spreading which might induce secondary abscess formation and bacteraemia. ${ }^{21}$ There are still several issues regarding extra corporeal shockwave therapy that have to be addressed, such as, high versus low energy extra corporeal shockwaves, shockwave dosage and number of sessions required for a therapeutic effect. Long term studies and results need to be awaited to be able to compare extra corporeal shockwave therapy with already established methods.

\section{REFERENCES}

1. Prabhuji M, Khaleelahmed S,Vasudevalu S, Vinodhini K Extracorporeal shock wave therapy in periodontics: A new paradigm. J Ind Soc Periodontol 2014 ;18,(3) 412-15

2. Gerdesmeyer L, Maier M, Haake M, Schmitz C. Physicaltechnical principles of extracorporeal shockwave therapy (ESWT). Orthopade 2002;31:610-7

3. Shrivastava SK, Kailash. Shock wave treatment in medicine. J Biosci 2005;30:269-75

4. Mittermayr R, Hartinger J, Antonic V, Meinl A, Pfeifer S,Stojadinovic . Extracorporeal shock wave therapy (ESWT) minimizes ischemic tissue necrosis irrespective of application time and promotes tissue revascularization by stimulating angiogenesis. Ann Surg 2011;253:1024-32

5. Escudier MP, Brown JE, Drage NA, McGurk M. Extracorporeal shockwave lithotripsy in the management of salivary calculi. Br J Surg 2003;90:482-5

6. Wang CJ, Wang FS, Yang KD. Biological effects of extracorporeal shockwave in bone healing: A study in rabbits. Arch Orthop Trauma Surg 2008; 128: 879-84

7. Muller P, Guggenheim B, Attin T, Marlinghaus E, Schmidlin PR. Potential of shockwaves to remove calculus and biofilm. Clin Oral Investig 2011; 15:959-965

8. Hazan-Molina H, Reznick AZ, Kaufman H, Aizenbud D. Assessment of IL-1 $\beta$ and VEGF concentration in a rat model during orthodontic tooth movement and extracorporeal shockwave therapy. Arch Oral Biol 2013; 58:142-150

9. Mariotto S, Cavalieri E, Amelio E, Ciampa AR, de Prati AC, Marlinghaus E. Extracorporeal shock waves: From lithotripsy to anti-inflammatory action by NO production. Nitric Oxide 2005; 12: 89-96

10. Wang FS, Yang KD, Chen RF, Wang CJ, Sheen Chen SM. Extracorporeal shock wave promotes growth and differentiation of bone-marrow stromal cells towards osteoprogenitors associated with induction of TGF-1. J Bone Joint Surg 2002; 84: 457-461

11. Mariotto S, de Prati AC, Cavalieri E, Amelio E, Marlinghaus E, Suzuki H. Extracorporeal shock wave therapy in inflammatory diseases: Molecular mechanism that triggers anti inflammatory action. Curr Med Chem 2009;16:23662372.

12. Sathishkumar S, Meka A, Dawson D, House N, Schaden W, Novak MJ. Extracorporeal shock wave therapy induces alveolar bone regeneration. J Dent Res 2008; 87: 687-691.

13. Gollwitzer H, Roessner M, Langer R. Safety and effectiveness of extracorporeal shockwave therapy: results of a rabbit model of chronic osteomyelitis. Ultrasound Med Bio 2009; 35(4): 595-602 
14. Wang CJ, Yang YJ, Huang CC. The effects of shockwave on systemic concentrations of nitric oxide level, angiogenesis and osteogenesis factors in hip necrosis. Rheumatol Int 2011; 31:871-877

15. Wang CJ, Wang FS, Yang KD, Weng LH, Hsu CC. Shock wave therapy induces neovascularization at the tendon-bone junction. A study in rabbits. J Orthop Res 2003;21:984-989

16. Novak KF, Govindaswami M, Ebersole JL, Schaden W, House N, Novak MJ. Effects of low-energy shock waves on oral bacteria. J Dent Res 2008; 87: 928-31

17. Hazan-Molina H, Kaufman H, Reznick ZA, Aizenbud D. Orthodontic tooth movement under extracorporeal shock wave therapy: The characteristics of the inflammatory reaction: A preliminary study. Refuat Hapeh Vehashinayim $2011 ; 28: 55-60$
18. Gerdesmeyer L, von Eiff C, Horn C, Henne M, Roessner M,Diehl P. Antibacterial effects of extracorporeal shock waves. Ultrasound Med Biol 2005; 31: 115-119

19. Li X, Chen M, Li L, Qing H, Zhu Z. Extracorporeal shock wave therapy: A potential adjuvant treatment for periimplantitis. Med Hypotheses 2010; 74: 120-122

20. Kerfoot WW, Beshai AZ, Carson CC. The effect of isolated high energy shock wave treatments on subsequent bacterial growth. Urol Res 1992; 20: 183-186

21. Xiao-feng Chen a, Xiao-lin Li b. Extracorporeal shock wave therapy could be a potential adjuvant treatment for orthopaedic implant-associated infections Journal of Medical Hypotheses and Ideas 2013; 7: 54-58 